\title{
Quantifying the Independence of Human Finger Movements: Comparisons of Digits, Hands, and Movement Frequencies
}

\author{
Charlotte Häger-Ross ${ }^{1,2}$ and Marc H. Schieber ${ }^{1}$ \\ ${ }^{1}$ Departments of Neurology, Neurobiology and Anatomy, and Brain and Cognitive Science, the Center for Visual Science, \\ and the Brain Injury Rehabilitation Program at St. Mary's Hospital, University of Rochester School of Medicine and \\ Dentistry, Rochester, New York, and 'Department of Community Medicine and Rehabilitation, Umeå University, SE-901 87 \\ Umeå, Sweden
}

To determine whether other digits move when normal humans attempt to move just one digit, we asked 10 right-handed subjects to move one finger at a time while we recorded the motion of all five digits simultaneously with both a video motion analysis system and an instrumented glove. We quantified the independence of the digits to compare (1) the different digits, (2) the right versus the left hand, and (3) movements at a self-paced frequency versus externally paced movements at $3 \mathrm{~Hz}$. We also quantified the degree to which motion occurred at the proximal, middle, or distal joint of each digit. Even when asked to move just one finger, normal human subjects produced motion in other digits. Movements of the thumb, index finger, and little finger typically were more highly individuated than were movements of the middle or ring fingers. Fingers of the dominant hand were not more independent than were those of the nondominant hand. Self-paced movements made at $\sim 2 \mathrm{~Hz}$ were more highly individuated than were externally paced movements at $3 \mathrm{~Hz}$. Angular motion tended to be greatest at the middle joint of each digit, with increased angular motion at the proximal and distal joints during $3 \mathrm{~Hz}$ movements. Simultaneous motion of noninstructed digits may result in part from passive mechanical connections between the digits, in part from the organization of multitendoned finger muscles, and in part from distributed neural control of the hand.

Key words: digits; human; hand; handedness; independence; individuation; fingers; laterality; movements; movement frequency; motor control
The digits of the human hand often are assumed to move independently of one another, like the digits of a robotic hand. Casual observation suggests, however, that in performing functional tasks humans rarely move one finger alone. Recordings of finger movements during grasping, typing, or piano playing reveal that multiple digits actually are in motion simultaneously. The degree of simultaneous motion depends on the behavioral task performed, however. In grasping objects of various sizes and shapes, high degrees of covariation have been observed among the metacarpophalangeal (MCP) joint angles of the four fingers and among the four proximal interphalangeal (PIP) joint angles as well (Santello and Soechting, 1998; Santello et al., 1998). To a lesser extent, simultaneous motion of multiple digits also occurs even during the single keystrokes of typing or piano playing (Fish and Soechting, 1992; Soechting and Flanders, 1992; Engel et al., 1997), because subjects have no specific requirement to keep the other fingers still while one finger strikes a key, as long as the other digits do not strike other keys. We therefore evaluated the ability of normal human subjects to move each digit independently when specifically asked to move one digit at a time, while not moving any other digits.

We also investigated three additional aspects of finger independence. First, because the phenomenon of handedness might be related to the independence of finger movements in the dominant versus nondominant hand, we compared the finger independence of normal subjects' right and left hands. Second, because the primary motor cortex (M1) is crucial for the production of individuated finger movements (Schieber and Poliakov, 1998) and because functional activation of the M1 hand representation has been found recently to increase with movement frequency (Rao et al.,

\footnotetext{
Received May 26, 2000; revised Aug. 16, 2000; accepted Aug. 24, 2000.

This work was supported by the National Institutes of Health Grant P41-RR0283. C.H.-R. was supported by a postdoctoral fellowship from the Swedish Medical Research Council.

Correspondence should be addressed to Dr. Marc H. Schieber, University of Rochester Medical Center, Department of Neurology, 601 Elmwood Avenue, Box 673, Rochester, NY 14642. E-mail: mhs@cvs.rochester.edu.

Copyright (C) 2000 Society for Neuroscience $0270-6474 / 00 / 208542-09 \$ 15.00 / 0$
}

1996; Sadato et al., 1996; Schlaug et al., 1996; Kawashima et al., 1999), we investigated whether finger independence is affected by movement frequency. And third, because our subjects were free to move the proximal, middle, or distal joint of each digit, we examined how normal subjects chose to distribute angular motion proximodistally across the three joints of each digit.

Parts of this paper have been published previously (Hager-Ross and Schieber, 1999).

\section{MATERIALS AND METHODS}

Subjects

Subjects with hands measuring at least $18 \mathrm{~cm}$ from the distal crease of the wrist to the end of the middle fingertip and with no previous medical history of trauma or degenerative or neurological disease affecting the upper limbs were recruited from hospital staff. Ten right-handed subjects (four men; six women; mean age, 32.6 years; range, 24-45 years) participated after giving written informed consent according to the Declaration of Helsinki. When asked whether they had any particular finger skill (such as typing) as a result of work or leisure activity, all subjects answered no. The study protocol was approved by the Research Subjects Review Board of the University of Rochester Medical Center and the Institutional Review Board of the Unity Health System (Rochester, New York). Each subject completed the 10 point Edinburgh Inventory to quantify their handedness (Oldfield, 1971) on a +100 (maximally right-handed) to -100 (maximally left-handed) laterality quotient scale. The laterality quotient for these 10 subjects ranged from +75 to +100 , with a mean of +89 .

\section{Experimental procedure}

The size and shape of the hand vary among normal human subjects. To compare the finger movements of different subjects, we therefore attempted to standardize the movements performed by each subject to the features of that subject's hands. The subject placed his/her right hand palm down on a piece of paper and abducted the digits as far as comfortable. In this position, the subject's hand was traced, and the tracing was used to create a guide for that subject's finger movements (Fig. 1 $A$ ) as follows. For each finger, a rectangle was drawn along the long axis of the traced finger, from the level of the MCP joint to the level of the distal interphalangeal (DIP) joint. For the thumb, a line was drawn from the thumb MCP joint to the web space between the middle and ring fingers, and a rectangle was drawn along this line from the long axis of the thumb to the long axis of the index finger. The pattern of these five rectangles was transferred to a piece of black cardboard, and corresponding rectangular slots were cut in 


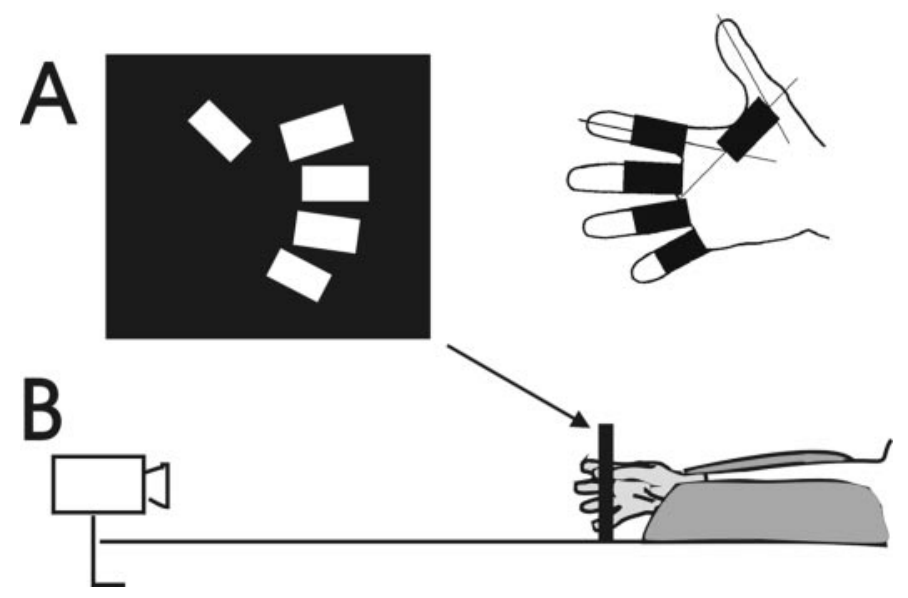

Figure 1. Schematic drawing of the experimental setup. A, Slots proportional to finger length (right) were cut in a piece of black cardboard (left) that then was placed in a vertical frame (arrow). When the subject placed each fingertip in the center of its own slot (as in $B$ ), these slots made the range of flexion-extension movements proportional to the size of each subject's fingers. $B$, Finger movements were recorded simultaneously with (1) a video camera mounted orthogonal to the plane of the cardboard frame (left) and (2) an instrumented glove worn by the subject (right).

the cardboard. The slotted cardboard was mounted in a fixed vertical frame to serve as a guide for that subject's finger movements (Fig. $1 B$ ). When the subject subsequently flexed and extended each finger within its individually prepared slot, the distance from the inner end (the point touched by flexing each digit) to the outer end (the point touched by extending each digit) was approximately equal to the distance from that finger's MCP joint to its DIP joint. The same slotted cardboard was flipped and reinserted in the frame to guide either the right-hand or left-hand fingers.

The subject then donned an instrumented glove and sat comfortably with the shoulder abducted $\sim 30^{\circ}$, the elbow joint flexed to $\sim 120^{\circ}$, and the forearm extended anteriorly in intermediate pronation/supination. The fingertips of the gloved hand were inserted into the appropriate cardboard slots with the fingers resting semiflexed. The forearm was positioned with the wrist extended $10-20^{\circ}$ to optimize the ability to perform finger movements (Hunter, 1990) and such that the tips of the fingers came to lie approximately in the center of the appropriate slots. The forearm and wrist were stabilized in a vacuum cast molded to the individual's forearm. Because pilot studies indicated no difference in performance depending on whether or not the hand was visible to the subject (Hayes and Schieber, 1996), the subject was allowed visual feedback of the hand to minimize inadvertent drifting of the fingers from the centers of the slots.

The subject was instructed to perform cyclical flexion-extension movements of one finger at a time, moving the instructed digit back and forth between the inner and outer edges of its slot. Movement of each digit was initiated when the subject was given a verbal instruction such as, "Now move your middle finger." After the experimenter verified that the subject was moving the correct digit, a manual switch was thrown generating a signal that marked the beginning of a period for data analysis; $3.5 \mathrm{sec}$ later a tone sounded signaling the end of the data analysis period. After hearing this tone the subject stopped cyclical movement of the instructed digit and awaited the next instruction. All subjects were naive to the task but performed one introductory series of movements of the different fingers before data collection. During data collection, instructed movements of the different fingers were performed in a pseudorandom order, with two epochs of instructed movement of each finger included in each recording. Two such recordings were made, providing in total four epochs of instructed movement of each finger. After completing these movements at the subject's self-paced frequency, the subject performed another four epochs of instructed movement of each digit, paced by a metronome at 3 $\mathrm{Hz}$. The subject performed both self-paced and $3 \mathrm{~Hz}$ movements first with one hand and then with the other, the right or left order being varied between subjects.

\section{Data acquisition}

As the subject performed the above tasks, movements of the fingers were recorded simultaneously with two complementary systems. First, a video recording was made at 60 frames/sec with a camera (JVC TK-1280) mounted $111 \mathrm{~cm}$ from the plane of the slotted cardboard guide to view the motion of the fingertips end on. To optimize the video image for automated off-line tracking of fingertip position (Motus, version 3.1; Peak Performance, Englewood, CO), a hemispherical reflective marker $(8 \mathrm{~mm}$ diameter) surrounded by a dark brown sheath of soft cotton was sewn onto each fingertip of the instrumented glove. The video system provided a veridical record of the flexion or extension motion of each fingertip projected orthogonally onto a two-dimensional plane.
A second system was used in parallel to record the motion of each finger joint. These recordings were made via an instrumented glove (mediumsize Cyberglove; Virtual Technologies, Palo Alto, CA), which was equipped with 22 resistive bend sensors that transduced motion of the joints of the hand. Data from each glove sensor were sampled at $54 \mathrm{~Hz}$ and stored to disk on a personal computer. Our analyses (below) used data from only 15 of the 22 sensors: the MCP, PIP, and DIP sensors for each finger (12 sensors) and the MCP, PIP, and opposition (OPP) sensors for the thumb ( 3 sensors). Calibration of the MCP, PIP, and DIP sensors for each digit was obtained for each subject by having the subject hold six objects of different size and shape. While the subject held each object, data were sampled from the glove, and the corresponding MCP, PIP, and DIP joint angles of each digit were measured with a hand-held goniometer. Plots of sensor reading versus joint angle typically were linear, and therefore linear regression was used to estimate the relationship between sensor output and joint angle. Pilot studies demonstrated that the OPP sensor provided output linearly related to motion of the carpometacarpal (CMC) joint in the flexion or extension plane of the thumb's three phalanges, and we therefore used the output of this sensor as a measure of motion at the $\mathrm{CMC}$ joint. Because we were unable to calibrate this sensor for each subject, however, the default calibration provided by the manufacturer was used for all subjects. Selecting subjects with a minimum hand size of $18 \mathrm{~cm}$ (above) ensured that the glove fit snugly enough to transduce the motion of most joints accurately, although in some subjects transduction of DIP motion was suboptimal.

\section{Data analysis}

Video recordings. Tracking fingertip positions in the video recordings demonstrated that the motion of each fingertip in the two-dimensional plane of the video image was constrained by the slots in the cardboard guide to be approximately linear (Fig. $2 A$ ). For each digit, we therefore calculated the best-fit line for its position throughout the recording, projected its position in each video frame onto that line, and normalized its position along the line from 1 at maximum flexion to 0 at maximum extension. This provided a one-dimensional measure of the normalized position of each digit within its own flexion-extension range. Plotting the normalized position of each digit as a function of time (Fig. $2 B$ ) then revealed that whereas instructed movements of some digits were accompanied by little if any movement of noninstructed digits, instructed movements of other digits were accompanied by considerable movement of noninstructed digits. For example, in the recording from subject 9 shown in Figure $2 B$, instructed movements of the thumb were accompanied by no detectable motion in other digits, but instructed movements of the ring finger were accompanied by overt motion in the middle and little fingers.

To quantify how much the other, noninstructed digits moved during a given instructed movement, we plotted the normalized position of each digit as a function of the simultaneous position of the instructed digit Figure $2 C$ shows an example of such a plot using the data from the second epoch of instructed movement of the ring finger in Figure $2 B$. Plots of the motion of a given digit as a function of the instructed digit's motion typically had a large linear component. We therefore computed the best-fit line for each such relationship and used the slope of that line as a measure of the relative motion of that noninstructed digit during that instructed movement. This relative motion slope will be close to 0 if the noninstructed digit did not move during the instructed movement and closer to 1 the more the instructed digit moved along with the instructed digit. In Figure $2 C$, for example, the plot of thumb position versus ring finger position has a slope close to 0 , reflecting the fact that the thumb did not move during instructed movement of the ring finger. The plot of little finger position versus ring finger position, however, has a positive, nonzero slope of 0.3 , reflecting the motion of the little finger during the same instructed movement.

We then used these relative motion slopes to derive two indexes, quantifying two aspects of the independence of each digit (Schieber, 1991). An ideally independent digit (1) would move when instructed with no accompanying movement of other digits and (2) would not move during instructed movement of other digits. To quantify how much the other, noninstructed digits moved during instructed movement of a given digit, we computed an individuation index (II) as 1 minus the average relative motion slopes of the noninstructed digits or:

$$
\mathrm{II}_{j}=1-\left[\left(\sum_{i=1}^{n}\left|S_{i j}\right|\right)-1\right] /(n-1),
$$

where $\mathrm{II}_{j}$ is the individuation index for instructed movement of the $j$ th digit, $S_{i j}$ is the slope of the relative motion of the $i$ th digit during the $j$ th instructed movement, and $n$ is the number of digits (here $n=5$ ). One is subtracted from the sum of the slopes in the numerator and from $n$ in the denominator to remove the slope of the instructed digit plotted against itself. The individuation index will be close to 1 for an ideally individuated movement in which the instructed digit moves with no movement of noninstructed digits and closer to 0 the more noninstructed digits move simultaneously with the instructed digit.

To quantify how much a given digit moves whenever it was a nonin- 

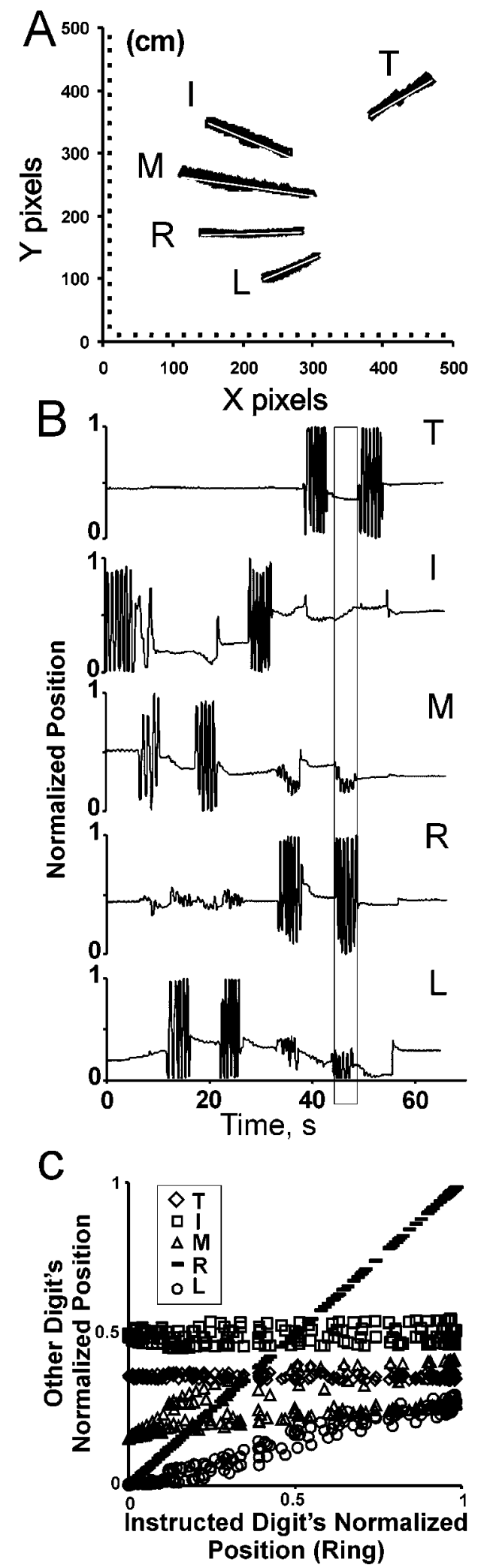

Figure 2. A, Fingertip positions throughout one recording are shown as viewed by the video system in a plane parallel to the slotted cardboard. The motion of the fingertips in this plane was essentially linear, because abduction and adduction movements were restricted by the slots. Linear regression was used to compute a best-fit line for each fingertip's positions (thin white lines). Scales are in video pixels; small squares inside the axes represent centimeters $(\mathrm{cm})$ at the fingertips. Data are from subject 9. B. The normalized position of each fingertip is shown as a function of time. All the data points for each fingertip in $A$ were projected onto the best-fit line for that finger, normalized from 0 (maximum extension) to 1 (maximum flexion) for each digit, and plotted as a function of time. $C$, Relative motion slopes are shown. Primarily linear relationships were evident when the normalized structed digit, we computed a stationarity index (SI) as 1 minus the average relative motion slope of that digit whenever it was a noninstructed digit or:

$$
\mathrm{SI}_{i}=1-\left[\left(\sum_{j=1}^{m}\left|S_{i j}\right|\right)-1\right] /(m-1),
$$

where $\mathrm{SI}_{i}$ is the stationarity index for the $i$ th digit during the $m$ instructed movements (here $m=5$ ). One is subtracted from the sum of the slopes in the numerator and from $m$ in the denominator to remove the slope of the noninstructed digit plotted against itself as the instructed digit. The stationarity index will be close to 1 for a digit that remains stationary whenever it is a noninstructed digit and closer to 0 the more the digit moves when it is a noninstructed digit.

Joint angle recordings from the instrumented glove. The joint angle recordings from the instrumented glove enabled us to examine how motion was distributed among the three joints of each digit. These analyses were performed for the three joints of each digit using data recorded during instructed movement of that digit. We plotted the angular position of a given joint against the angular position of each of the three joints in the same digit. These plots typically were relatively linear, indicating that subjects used a relatively constant ratio of angular movement among the three joints of a given digit. We therefore computed the slope of the best-fit line for the relationships of a given joint plotted as the dependent variable against each of the three joints plotted as the independent variable, which we call the relative angular motion slope. For the given joint plotted against itself, this slope of course was 1 . The relative angular motion slope approached 0 the less the given joint moved compared with another joint of the same digit, but the slope reached values much larger than 1 if the given joint moved much more than the other joint. For each joint, we then totaled the absolute values of the three relative angular motion slopes obtained when the angular position of that joint was used as the dependent variable. This total was close to 1 if the given joint moved very little during instructed movement of the digit and was larger the more the joint moved.

We then used the relative angular motion slopes to derive two indexes quantifying the proximodistal distribution of angular motion in the joints of each digit (cf. Fritz et al., 1992). Computation of these indexes is simplified by expressing the total relative angular motion slope of each joint as a fraction of the sum across the three joints. If $T_{i}$ is the total relative angular motion slope of the $i$ th joint, then the fractional total $\tau_{i}$ is:

$$
\tau_{i}=T_{i} / \sum_{i=1}^{n} T_{i},
$$

where $n$ is the number of joints (here $n=3$ ). We then computed a proximodistal index (PDI) to quantify the proximodistal distribution of joint rotation from -1 to +1 , with +1 representing all angular motion occurring at the most proximal joint, 0 representing angular motion distributed symmetrically about the middle joint, and -1 representing all angular motion occurring at the most distal joint. The PDI is calculated as:

$$
\mathrm{PDI}=\sum_{i=1}^{n} \tau_{i}^{*} w_{i}
$$

where $\tau_{i}$ is the fractional total relative angular motion slope of the $i$ th joint, $n$ is the number of joints, and $w_{i}$ is a constant that provides a rank-ordered weighting of the joints:

$$
w_{i}=(2 i-n-1) /(n-1) .
$$

We also computed a divergence index (DIV) to quantify the degree to which angular motion occurred at just one joint $(\mathrm{DIV}=0)$ versus being spread evenly over the joints (DIV $=1)$. The DIV is calculated as:

$$
\mathrm{DIV}=s^{*} \sum_{i=1}^{n} \tau_{i}^{*}\left(\mathrm{PDI}-w_{i}\right)^{2},
$$

where $s$ is a scaling factor that normalizes for the number of joints:

$$
s=3^{*}(n-1) /(n+1) \text {. }
$$

Statistical analyses. In addition to descriptive statistics, separate tests of ANOVA with a nested design were used to determine main effects of

position of each digit was plotted against the simultaneous normalized position of the instructed digit throughout flexion-extension cycles of a given instructed movement. We used the slopes of these relationships to quantify the relative motion of each digit during instructed movement of a given digit. Data shown represent the period of instructed ring finger movement indicated by the vertical rectangle in $B$. I, Index; $L$, little; $M$, middle; $R$, ring; $T$, thumb. 
Table 1. Individuation indexes during self-paced movements

\begin{tabular}{llllll} 
& \multicolumn{2}{l}{$\begin{array}{l}\text { Individuation index of right } \\
\text { hand }\end{array}$} & & \multicolumn{2}{l}{$\begin{array}{l}\text { Individuation index of left } \\
\text { hand }\end{array}$} \\
\cline { 2 - 3 } Digit & Mean \pm SD & Range & & Mean \pm SD & Range \\
\hline Thumb & $0.983 \pm 0.013$ & $0.947-0.999$ & & $0.991 \pm 0.006$ & $0.976-0.998$ \\
Index & $0.982 \pm 0.012$ & $0.951-0.995$ & & $0.974 \pm 0.022$ & $0.928-0.999$ \\
Middle & $0.937 \pm 0.034$ & $0.860-0.992$ & & $0.935 \pm 0.039$ & $0.851-0.995$ \\
Ring & $0.907 \pm 0.046$ & $0.809-0.985$ & & $0.898 \pm 0.060$ & $0.735-0.985$ \\
Little & $0.943 \pm 0.042$ & $0.825-0.994$ & & $0.945 \pm 0.040$ & $0.836-0.996$
\end{tabular}

subject (1-10), hand (right or left), and finger (1-5) on the following dependent variables: (1) individuation index, (2) stationarity index, (3) proximodistal index, and (4) divergence index. To test for differences between the two conditions with different movement frequency (self-paced vs externally paced at $3 \mathrm{~Hz}$ ), we used two-way ANOVA (frequency and finger as independent factors). For each test the level of probability chosen as statistically significant was $p<0.05$. Nonparametric correlation was done using the Spearmans' rank correlation test, and the Wilcoxon matched-pairs signed-ranks test was used to evaluate differences pairwise between fingers on the right and left hand. Bonferroni corrections on the significance level were implemented to compensate for the number of statistical tests. Group estimates are presented in the form of means \pm SD values unless otherwise stated in the text.

\section{RESULTS}

\section{Individuation of human finger movements}

An ideally independent finger would move without any accompanying motion of the other fingers. As illustrated in Figure 2, however, when instructed to move only one finger, human subjects often move other fingers as well. The extent of motion in noninstructed digits varied from finger to finger and depended on which finger was instructed to move. We used an individuation index to quantify the degree to which noninstructed digits moved during instructed movement of a given digit. The individuation index varies from 1 if there is no motion of any noninstructed digit to 0 if the noninstructed digits all move as much as the instructed digit (see Materials and Methods).

Table 1 presents the mean, SD, and range of the individuation indexes for self-paced movements of each finger, averaged across subjects. As might be expected, in both the right hand and left hand the thumb had the highest average individuation index. The index finger likewise had a high average individuation index, very close to that of the thumb. The little finger ranked third on average, whereas the middle finger and especially the ring finger tended to have the lowest individuation indexes. When the individuation indexes for the different digits of each hand of each subject were rank ordered from 1 (highest) to 5 (lowest), averaging across hands and subjects gave mean rankings for the thumb of 1.60 , index finger of 1.60, middle finger of 3.63, ring finger of 4.65, and little finger of 3.50. These values are consistent with the common experience that the thumb and index finger are the most independent digits and the middle and ring fingers are the least independent.

Such was not always the case for individual subjects, however. Figure 3 shows the individuation indexes for instructed movement of each digit of the right hand of the 10 subjects $(A)$ and of each digit of the left hand $(B)$ and compares the medians and quartiles for the right and left hands $(C)$. Each point in Figure 3, $A$ and $B$, represents the mean of values computed from the four different epochs of a given movement performed by each subject. Although thumb movements often were the most highly individuated for a given subject, approximately half of the subjects showed a slightly higher individuation index on average for instructed movements of the index finger. Even the other fingers occasionally showed comparably high individuation indexes in particular subjects. Ring finger movements clearly tended to have the lowest individuation indexes. For all subjects except one (subject 10) the ring finger had the lowest individuation index in the right hand, and for all subjects except three (subjects 5, 8, and 10) the ring finger had the lowest individuation index in the left hand.

\section{Stationarity of human fingers}

In addition to moving without any accompanying motion of the other fingers, an ideally independent finger would remain stationary during instructed movement of other digits. We used a stationarity index to quantify the degree to which a given digit remained still during instructed movements of the other digits (see Materials and Methods). The stationarity index for a given digit can vary from 1 , if there is no motion of that digit during instructed movement of any other digit, to 0 , if that digit moves as much as the instructed digit.

Table 2 presents the mean, SD, and range of the stationarity indexes for self-paced movements of each finger, averaged across subjects. These indexes indicate that, on average, the thumb remained most stationary during instructed movements of other fingers. The index finger and the little finger likewise remained relatively still when other fingers were moved, whereas the middle finger and particularly the ring finger tended to move the most and therefore had lower stationarity indexes. This rank order in the average stationarity index of the different digits did not apply consistently to the indexes for individual subjects, however. Figure

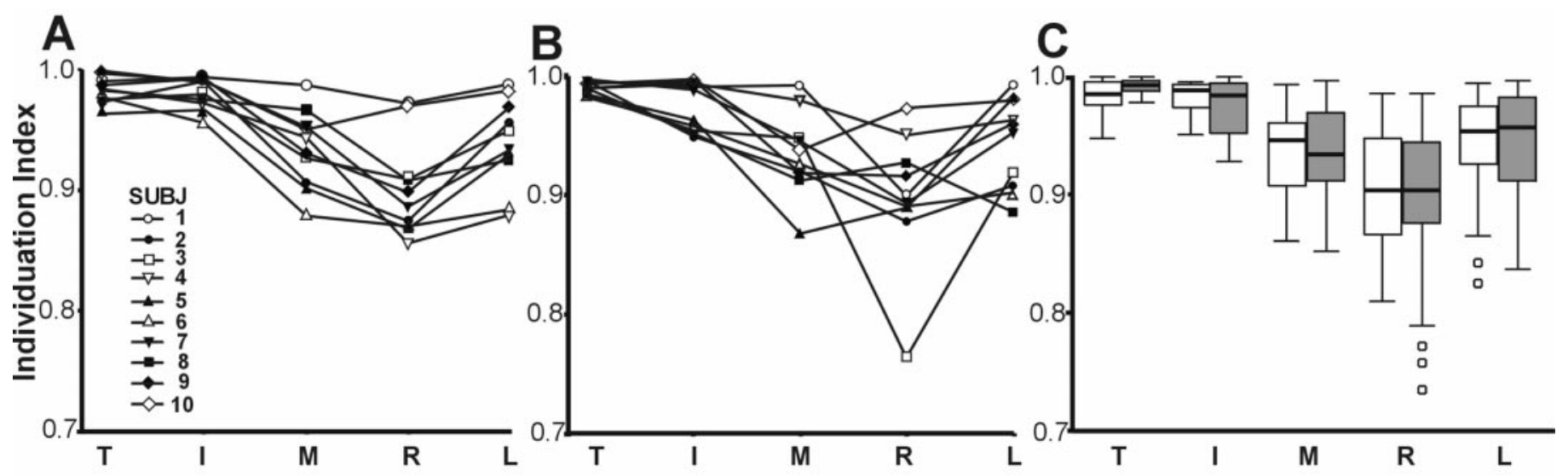

Figure 3. Individuation indexes during self-paced movements. $A, B$, Symbols connected by a line represent individuation indexes averaged over four epochs of instructed movements of the thumb $(T)$, index $(I)$, middle $(M)$, ring $(R)$, and little $(L)$ finger of each subject's right $(A)$ and left $(B)$ hands. $C$, Box plots comparing the distributions across subjects display the median and quartiles of all values of the individuation indexes for the right (white boxes) and left (shaded boxes) hands. Each box represents the 25th-75th percentile, and the horizontal line across the box is the median (50th percentile). Whisker lines extending above and below each box indicate the total range with the exception of small circles beyond the whiskers that represent outliers $>1.5$ box lengths away from the bottom or top of the box. Whereas the points shown in $A$ and $B$ each represent the average of values from four epochs of the same instructed movement, each of these four values contributed separately to the distributions shown in $C$. SUBJ, Subject. 
Table 2. Stationarity indexes during self-paced movements

\begin{tabular}{llllll} 
& \multicolumn{2}{l}{$\begin{array}{l}\text { Stationarity index of right } \\
\text { hand }\end{array}$} & & \multicolumn{2}{l}{ Stationarity index of left hand } \\
\cline { 2 - 3 } Digit & Mean \pm SD & Range & & Mean \pm SD & Range \\
\hline Thumb & $0.994 \pm 0.005$ & $0.983-1.000$ & & $0.996 \pm 0.005$ & $0.974-0.999$ \\
Index & $0.967 \pm 0.021$ & $0.891-0.997$ & & $0.973 \pm 0.016$ & $0.935-0.996$ \\
Middle & $0.941 \pm 0.031$ & $0.864-0.991$ & & $0.925 \pm 0.048$ & $0.808-0.992$ \\
Ring & $0.908 \pm 0.054$ & $0.755-0.982$ & & $0.905 \pm 0.052$ & $0.811-0.996$ \\
Little & $0.943 \pm 0.036$ & $0.824-0.992$ & & $0.943 \pm 0.035$ & $0.864-0.996$
\end{tabular}

4 shows that, as with the individuation index, the stationarity indexes of the middle and ring fingers showed the greatest variability among subjects.

Nevertheless, digits with high individuation indexes tended to have high stationarity indexes as well. Indeed, within a hand, the different digit's individuation and stationarity indexes were significantly correlated $\left(r_{s}=0.74 ; p<0.01\right.$; Spearmans' rank correlation; values pooled across hands and subjects). This suggests that similar factors both enable a specific finger to move without movement in the other fingers and enable that finger to remain still while other fingers move.

\section{Are the fingers of the dominant hand more independent than those of the nondominant hand?}

Introspection suggests that the fingers of one's dominant hand might be more independent than those of the nondominant hand. We used our data to test this hypothesis. Figure 3 shows that although some subjects had slightly higher individuation indexes for their dominant, right fingers (Fig. $3 A$ ) than for their nondominant, left fingers (Fig. $3 B$ ), the group median and quartiles for each digit of the right and left hands were quite similar (Fig. 3C), as were their means and SDs (Table 1). An ANOVA-nested design (subject, hand, and finger), although confirming a clear main effect of finger $(80, F=19.4 ; p<0.0001)$, showed no significant main effect of hand $(10, F=0.6 ; p>0.80)$ on the individuation index. Performing a separate paired comparison of the individuation indexes for each digit of the right versus left hand revealed a significant difference only for the thumb, where the individuation index was higher for the left, nondominant thumb than for the right $(p<0.001$ after Bonferroni correction for five tests, Wilcoxon matched pairs). Similarly, although some subjects had higher stationarity indexes for the dominant hand (Fig. 4, $A$ vs $B$ ) and although for the group of 10 subjects the stationarity indexes varied significantly across the five digits $(80, F=18.3 ; p<0.001$, nested
ANOVA), stationarity indexes were not significantly different in the right versus left hands $(10, F=0.6 ; p>0.78)$. In the present task, the independence of the digits thus does not appear to differ systematically between the dominant and nondominant hands.

\section{Effects of movement frequency}

When asked to perform cyclical flexion-extension movements at a comfortable pace, our subjects chose frequencies of $\sim 2 \mathrm{~Hz}$ on average for each digit (Table 3 ). The measured movement frequencies of the middle and ring fingers were generally lower compared with that of the other digits. Moreover, self-paced frequencies of the middle and ring fingers of the dominant, right hand tended to be lower than were those of the middle and ring fingers of the left hand, although these differences were not significant after Bonferroni correction. When the same subjects performed movements externally paced at $3 \mathrm{~Hz}$, the measured movement frequency was very close to $3 \mathrm{~Hz}$ for all digits of both hands (Table 3 ). Furthermore, the variability of movement frequency, as assessed by the group SDs, was reduced during externally paced movements at 3 $\mathrm{Hz}$ as compared with self-paced movements at $2 \mathrm{~Hz}$, especially for the left hand. Metronome pacing at $3 \mathrm{~Hz}$ thus increased the frequency and decreased the variability of the cyclical flexionextension movements performed by the subjects.

Externally paced movements at $3 \mathrm{~Hz}$ were clearly less independent than were self-paced movements at $2 \mathrm{~Hz}$. Reductions of the group individuation and stationarity indexes in the $3 \mathrm{~Hz}$ condition were evident for each digit of both the dominant hand and the nondominant hand (Fig. 5). As with the self-paced movements, there was no general effect by hand on the individuation or stationarity indexes during the $3 \mathrm{~Hz}$ condition $(10, F=0.8 ; p>0.6$; and $10, F=0.7 ; p>0.8$, respectively, ANOVA-nested design in both cases). Therefore we pooled the data from the right and left hands to compare the independence of externally paced movements at $3 \mathrm{~Hz}$ versus self-paced movements. Two-way ANOVA (frequency and finger) on these pooled data confirmed that individuation indexes $(1, F=46.7 ; p<0.001)$ and stationarity indexes $(1, F=49.9 ; p<0.001)$ both were significantly lower during externally paced movements at $3 \mathrm{~Hz}$.

The decrease in finger independence observed during externally paced movements at $3 \mathrm{~Hz}$ might have been related to the external pacing, the higher frequency, or both. To explore the effect of movement frequency on finger independence further, we examined the correlation between measured movement frequency and the individuation index during self-paced movements. Within- and between-subject variation provided a range of measured frequencies from $\sim 0.7$ to $3.3 \mathrm{~Hz}$ for self-paced movements. Because of the effect of digit on individuation index, we tested the correlation between the individuation index and measured movement fre-
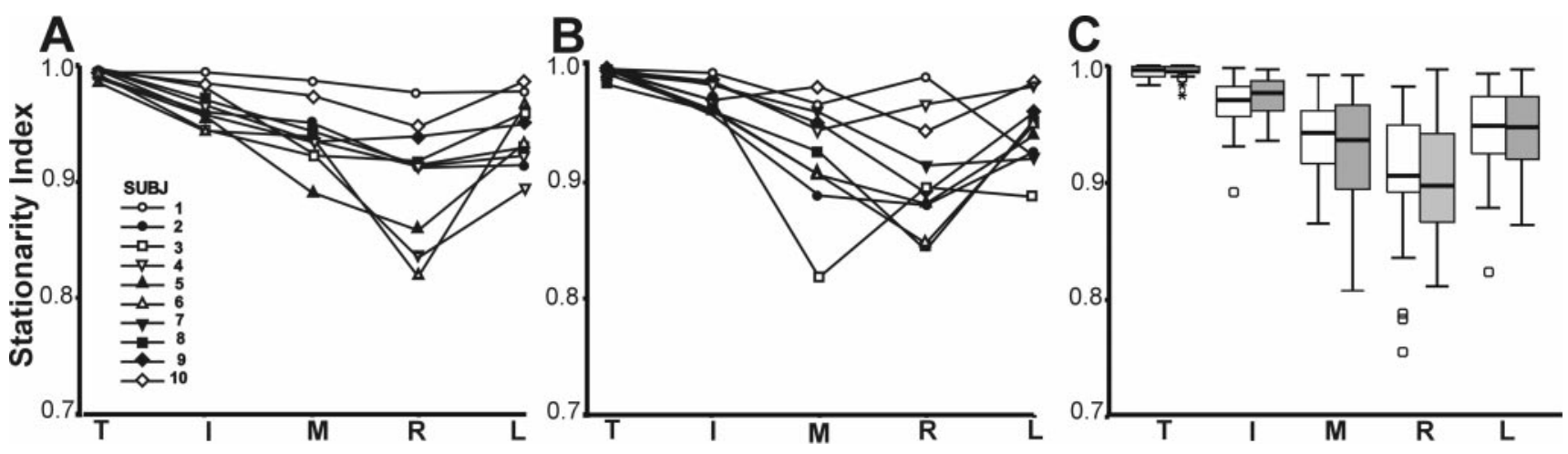

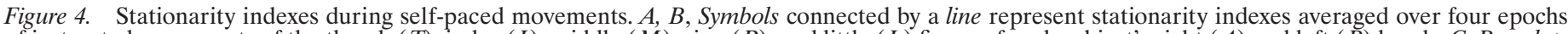

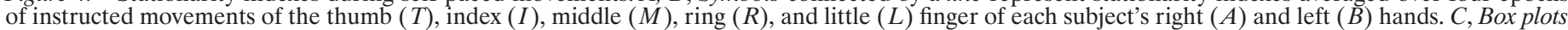

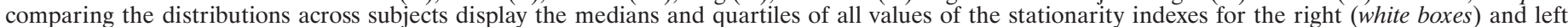

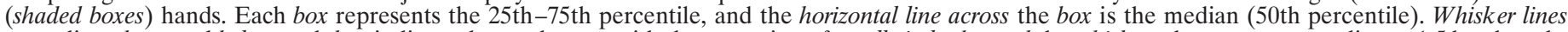

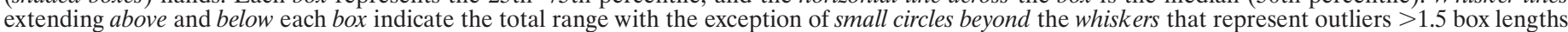

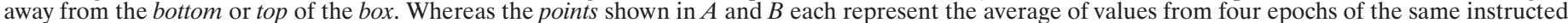
movement, each of these four values contributed separately to the distributions shown in $C$. SUBJ, Subject. 
Table 3. Measured movement frequencies

\begin{tabular}{llllll} 
& \multicolumn{2}{l}{ Self-paced } & & & Externally paced at $3 \mathrm{~Hz}$ \\
\cline { 2 - 3 } Digit & Right $($ mean $\pm \mathrm{SD}), \mathrm{Hz}$ & Left $($ mean $\pm \mathrm{SD}), \mathrm{Hz}$ & & Right (mean $\pm \mathrm{SD}), \mathrm{Hz}$ & $\mathrm{Left}(\mathrm{mean} \pm \mathrm{SD}), \mathrm{Hz}$ \\
\hline Thumb & $2.10 \pm 0.61$ & $2.24 \pm 0.63$ & $2.99 \pm 0.36$ & $2.97 \pm 0.20$ \\
Index & $2.03 \pm 0.65$ & $2.20 \pm 0.58$ & $3.00 \pm 0.37$ & $3.00 \pm 0.11$ \\
Middle & $1.94 \pm 0.64$ & $2.19 \pm 0.62$ & $2.95 \pm 0.38$ & $3.02 \pm 0.12$ \\
Ring & $1.94 \pm 0.56$ & $2.17 \pm 0.62$ & $2.92 \pm 0.39$ & $3.94 \pm 0.22$ \\
Little & $2.17 \pm 0.67$ & $2.25 \pm 0.66$ & $3.02 \pm 0.42$ & \\
\hline
\end{tabular}

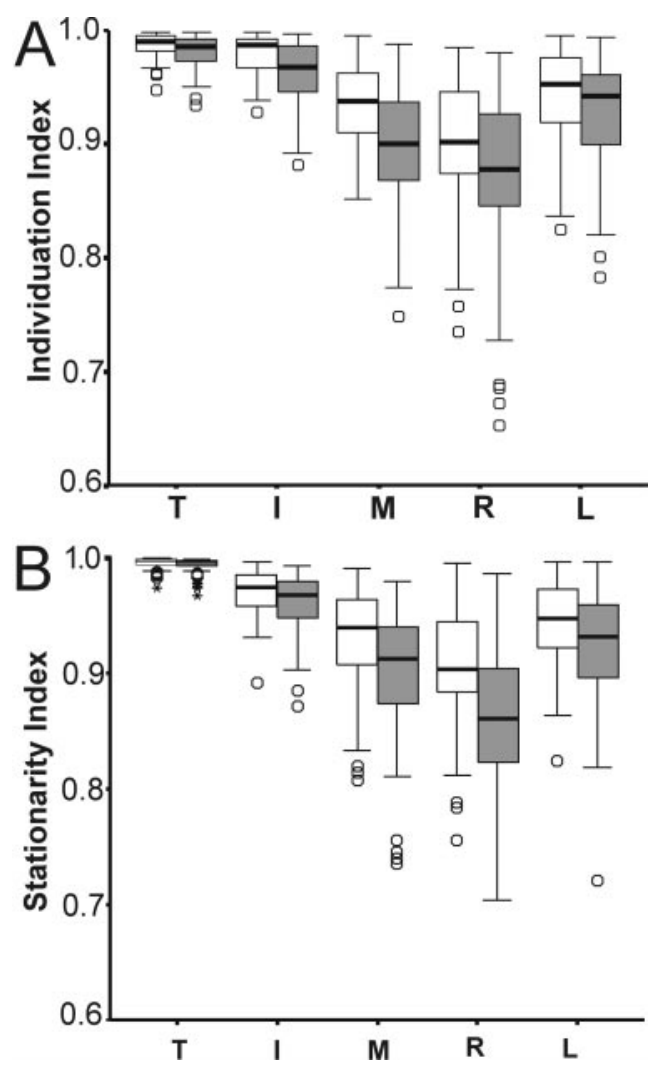

Figure 5. Box plots comparing the independence of movements performed at a self-paced frequency (white boxes) versus movements paced by a metronome at $3 \mathrm{~Hz}$ (shaded boxes). $A$, Individuation indexes. $B$, Stationarity indexes. Box plots are described in Figure 3. Note the consistently lower indexes during the metronome-paced movements at $3 \mathrm{~Hz}$. Data are pooled across both hands. $I$, Index; $L$, little; $M$, middle; $R$, ring; $T$, thumb.

quency separately for each digit, while pooling data values from each movement epoch across right and left hands and across subjects. Negative correlations-lower individuation indexes at higher movement frequencies-were found for all digits except the thumb. These correlations were significant $(p<0.05)$ for the four fingers, although the correlation for the middle finger was not significant after Bonferroni correction for five tests (uncorrected $p$ values, thumb $p=0.14$; index finger $p<0.001$; middle finger $p<$ 0.037 ; ring finger $p<0.001$; little finger $p<0.001)$. These observations suggest that movement frequency per se has an effect on individuation: movements performed at higher frequencies tending to be less individuated than are those performed more slowly.

\section{Interjoint coordination}

Data recorded with the instrumented glove enabled us to examine how the subjects moved the various finger joints. Figure 6 shows glove sensor data on the simultaneous angular motion of 15 finger joints during the two $3.5 \mathrm{sec}$ epochs of flexion-extension movements of each digit in a single recording from subject 5. Inspection of these data suggests that the distribution of angular motion across the three proximodistal joints varied from digit to digit. For the thumb, the least angular motion occurred at the CMC joint, the most at the MCP, and an intermediate amount at the PIP. For the middle finger, an intermediate amount of angular motion occurred at the MCP joint, the most at the PIP, and the least at the DIP. For the little finger, the most angular motion occurred at the MCP joint, an intermediate amount at the PIP, and the least at the DIP.

To permit comparison of the proximodistal distribution of angular motion in the different digits of the same subject, as well as across subjects, for the four epochs of each digit's movement recorded from each subject during self-paced movements, we calculated two complementary indexes. The PDI ranging from +1 to -1 summarizes the extent to which angular motion occurred at the digit's proximal, middle, or distal joint. The divergence index summarizes the degree to which motion occurred equally at all three joints $(\mathrm{DIV}=1)$ versus only at a single joint $(\mathrm{DIV}=0)$. Figure 7 displays the average of these values for each digit of each subject's right (Fig. $7 A$ ) and left (Fig. $7 B$ ) hands. For all digits of both hands, the DIV index had midrange values, indicating that motion occurred at multiple joints $(>0)$ but not equally at all joints $(<1)$. At which joints the motion occurred is indicated by the corresponding PDI values. For example, the PDI of +0.5 for the little finger of subject 5's right hand (Fig. 7A, Little, $\mathbf{\Delta}$ ) reflects that in this case the MCP joint moved more than did the PIP or DIP, as can be seen by inspection of Figure 6, bottom row. For the four fingers, considering the group of 10 subjects as a whole, PDI values skewed to the positive side of 0 indicate that the most angular motion occurred at the PIP joints followed by the MCP joints. For the thumb, the PDI values skewed to the negative side of 0 indicate that for the group as a whole the most angular motion occurred at the MCP (middle joint of the thumb), with less at the PIP (distal) and still less at the CMC joint (proximal). Thus for all five digits, the greatest angular motion occurred at the middle joint (PIP for the fingers and MCP for the thumb). The distribution of angular motion at the proximal and distal joints differed, however. The four fingers showed more motion at the proximal MCP than at the distal DIP $($ PDI $>0)$, whereas the thumb showed more motion at the distal PIP than at the proximal CMC joint (PDI $<0)$.

Figure 7 also reveals considerable variability. That the proximodistal distribution of joint motion differed among subjects was confirmed by a main effect on the PDI and DIV by subject $(9, \mathrm{~F}=$ $3.6 ; p<0.03$; and $9, \mathrm{~F}=5.2 ; p<0.008$, respectively, nested ANOVA). The PDI and DIV were similar for right and left hands, however $(10, F=0.3 ; p>0.98$; and $10, F=0.3 ; p>0.97$, respectively, nested ANOVA). Likewise, there was no effect on the PDI by the movement frequency $(1, F=2.6 ; p>0.1$, two-way ANOVA). The DIV, on the other hand, changed with movement frequency $(1, F=9.8 ; p<0.002$, two-way ANOVA): The DIV values were higher in the condition with externally paced movements at $3 \mathrm{~Hz}$, indicating that the movements were more distributed across all three joints in this condition.

\section{DISCUSSION}

\section{Independence of human finger movements}

We found that even when asked specifically to move one digit without moving any other digits, normal human subjects produced 


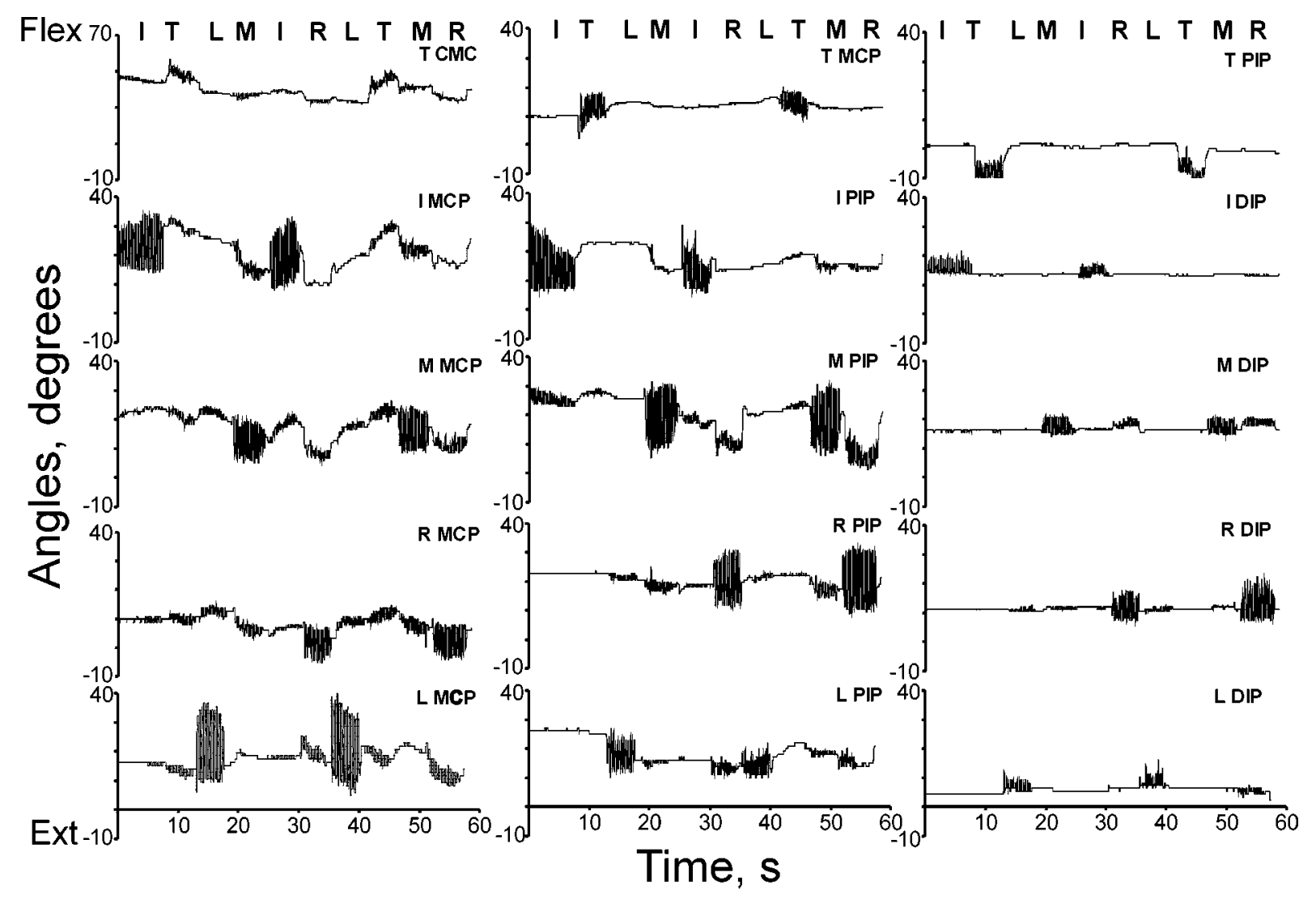

Figure 6. Data recorded simultaneously from 15 finger joint sensors of the instrumented glove for a single subject (number 5 ) during two 3.5 sec epochs of instructed flexion-extension movements for each digit. Although all data were recorded simultaneously, traces from different joints are shown in three columns representing the motion of the most proximal joints (left), the middle joints (center), and the most distal joints (right) of each digit, as indicated in the right corner above each trace. Rows of three traces thus represent the joints of each digit from the thumb at the top to the little finger at the bottom. The pseudorandom sequence of finger movements instructed to the subject is indicated above each column. CMC, Carpometacarpal; DIP, distal interphalangeal; $I$, index; $L$, little; $M$, middle; $M C P$, metacarpophalangeal; $P I P$, proximal interphalangeal; $R$, ring; $T$, thumb.

low-amplitude motion in noninstructed digits simultaneous with the large-amplitude motion of the instructed digit. Consistent with common experience, instructed movements of the ring and middle fingers were associated with the greatest motion of noninstructed digits, whereas the thumb and index finger showed the highest degrees of individuation and stationarity, i.e., the greatest independence.

Our findings resemble those obtained in isometric paradigms involving much higher muscular forces. When subjects were asked to flex one finger so as to exert maximal isometric force at the fingertips, at the DIP joints or at the PIP joints, forces exerted by other fingers ranged from 2 to $52 \%$ of the force exerted by the instructed finger (Zatsiorsky et al., 1998). Without actively extending the other fingers away from contact at the flexor surface, subjects were unable to exert high forces in one finger without exerting substantial forces in the others. Our results suggest that the same is true in the low range of internal muscular forces needed to flex and extend the unloaded fingers. Normal subjects cannot generate the forces needed to move one finger isotonically through a substantial range without generating forces that simultaneously move adjacent noninstructed digits through a smaller range.

Most humans are right-handed, preferring to perform fine manipulations with the right hand. The preferred hand feels easier to control in complex movements. Such ease of control might result from a greater independence of finger movements in the preferred hand. In strongly right-handed subjects, however, we found no evidence of a systematic difference in finger movement independence between the right and left hands in terms of either individuation or stationarity. Our results are consistent with previous studies in which movements of single fingers were scored by visual observation (Kimura and Vanderwolf, 1970; Parlow, 1978). This suggests that handedness is not related simply to a greater independence of finger movements in the preferred hand. Indeed, tests of performance that are sensitive to handedness emphasize the speed of fine, accurate movements involving multiple digits simultaneously rather than finger independence alone (Annett, 1992; Jancke, 1996). Physiological differences in motor units and motor cortical activation on the dominant versus nondominant side (Schmied et al., 1994; Dassonville et al., 1997; Adam et al., 1998; Semmler and Nordstrom, 1998; Triggs et al., 1999) may not result in a difference in finger independence per se.

In contrast, we found a systematic decrease in the independence of finger movements externally paced at $3 \mathrm{~Hz}$ compared with self-paced movements at $\sim 2 \mathrm{~Hz}$. Although we cannot exclude that the external pacing contributed to this difference, an effect of higher frequency seems most likely (Wessel et al., 1997). The greater motion of noninstructed digits during movements at higher frequency probably results in part from passive viscoelastic coupling between the digits; to cover the same distance at higher movement frequencies the instructed digit must be moved at higher velocity, increasing any velocity-dependent tendency to pull noninstructed digits along. To keep noninstructed movements as stationary as possible, however, the nervous system may generate additional muscle activity to counteract such viscoelastic forces, a function that in part may be mediated in the primary motor cortex (Humphrey and Reed, 1983). Greater functional activation of the M1 hand representation occurs during movements performed at higher frequencies (Rao et al., 1996; Sadato et al., 1996; Schlaug et al., 1996; Kawashima et al., 1999). Such frequency-dependent increases in M1 activity thus may result not only from the performance of more movement cycles per unit time and the production of higher muscular forces and rates of change of force to move 


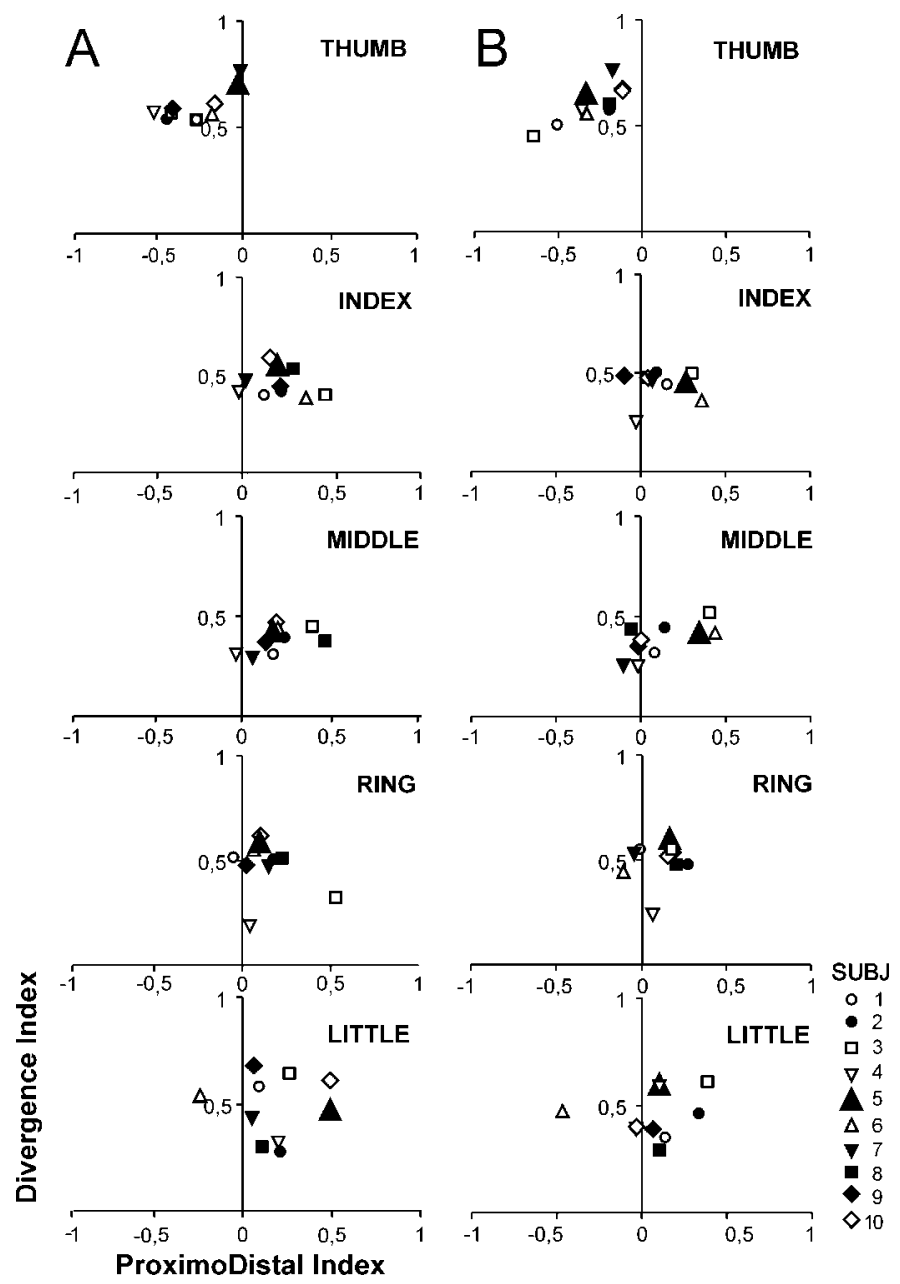

Figure 7. The DIV and PDI during self-paced movements. $A$, Right hand. $B$, Left hand. The DIV quantifies the extent to which the movements occurred in one joint versus being distributed across all three joints, whereas the PDI quantifies in which of the joints (MCP/CMC, PIP, or DIP) the motion mainly took place (see Materials and Methods). The figure displays mean data calculated over all four epochs of each instructed movement. The raw data from subject $5(\boldsymbol{\Lambda})$ for two of the four epochs of each instructed movement are shown in Figure 6.

through the same amplitude at higher velocities, but also from the additional muscular forces needed to stabilize noninstructed digits at higher movement frequencies (Schieber, 1990; Remy et al., 1994).

\section{Factors affecting finger independence}

The individuation and stationarity indexes measured in the present study varied considerably among our 10 normal human subjects. To some extent such intersubject variability may reflect differences in the long-term motor experiences of each subject. This variability also may result from intersubject differences in anatomic and physiological factors that affect the independence of the fingers, including biomechanical connections between the digits, functional organization of multitendoned finger muscles, and differences in the central inputs to spinal motoneuron pools.

Biomechanical interconnections between the digits come from many sources. The soft tissues of the web spaces couple adjacent fingers to some degree (von Schroeder and Botte, 1993). Better known are the juncturae tendinium of extensor digitorum communis (EDC) (Fahrer, 1981; von Schroeder et al., 1990). Just proximal to the MCP joints, these bands of connective tissue connect the EDC tendons running to adjacent fingers. In addition, the flexor digitorum prof undus (FDP) tendons to the middle, ring, and little fingers typically are interconnected as they arise from the muscle belly and may also be interconnected within the palm by the bellies of the interosseous muscles that take origin from two adjacent FDP tendons (Fahrer, 1981). Furthermore, 20-30\% of normal subjects may have "anomalous" interconnections, such as a tendinous band between flexor pollicis longus and the index finger portion of FDP (Linburg and Comstock, 1979; Austin et al., 1989; Gonzalez et al., 1997).

Finger movements also may be coupled by the organization of motor units in the multitendoned extrinsic finger muscles-FDP, flexor digitorum superficialis (FDS), and EDC. The extent to which these muscles contain a separate neuromuscular compartment acting on each finger versus compartments that act on multiple fingers simultaneously is an area of active investigation. In the extensor digitorum quarti et quinti of macaque monkeys (homolog of the human extensor digiti minimi), many single motor units have been found to act on both of the independent tendons to digits 4 and 5 (Schieber et al., 1997). In the human EDC, the tension of single motor units is distributed to multiple fingers more broadly than can be attributed to mechanical interconnections between the tendons (Keen and Fuglevand, 1999). In the present study, our subjects produced more motion at the PIP joint than at the MCP or DIP. We speculate that this may reflect a greater finger selectivity of motor units in FDS, which act across the MCP and PIP joints, compared with motor units in FDP, which act across the MCP, PIP, and DIP joints.

Finally, the motoneuron pools innervating different finger muscles receive considerable shared central input. As revealed by short-term synchronization between single motor units in different human muscles, intrinsic and extrinsic muscles acting on different fingers receive shared inputs (Bremner et al., 1991a,b). Shared central inputs have been shown in monkeys to come from spinal interneurons, rubrospinal neurons, and corticospinal neurons (Fetz and Cheney, 1980; Buys et al., 1986; Mewes and Cheney, 1991, 1994; Fetz et al., 1996; McKiernan et al., 1998; Perlmutter et al., 1998). Such shared inputs may account for the observation that as normal human subjects flex the tip of one finger, FDP motor units acting on adjacent fingers are recruited shortly after motor units acting on the finger being flexed (Kilbreath and Gandevia, 1994). Indeed, single neurons in the monkey M1 hand representation typically discharge during individuated movements of several different digits (Schieber and Hibbard, 1993; Poliakov and Schieber, 1999).

Human subjects demonstrated substantially higher individuation and stationarity indexes than those described previously in monkeys (Schieber, 1991). Species differences may shed additional light on finger independence. Pertinent differences may be found between the extrinsic multitendoned finger muscles of monkeys and humans (Serlin and Schieber, 1993). In macaques the mechanical interconnections among the tendons of FDP are more extensive than in humans, and the distal tendon to the thumb arises from FDP, with no separate flexor pollicis longus (FPL). Macaques also have two multitendoned finger muscles (extensor digitorum secundi et tertii and extensor digitorum quarti et quinti) that are homologous to monotendoned muscles in humans (extensor indicus proprius and extensor digiti quinti, respectively). These differences suggest that the greater independence of finger movements in humans arises in part from the splitting of a separate muscle belly and tendon in some instances (FPL) and from the loss of a tendon from multitendoned muscles in others (extensor indicis proprices and extensor digiti quinti), enhancing the independence of the thumb and index and little fingers in particular. Additional differences may exist in the M1 hand representation. Although humans and monkeys both show extensive overlap of the M1 territory activated during movements of different fingers (Schieber and Hibbard, 1993; Sanes et al., 1995), humans may have a somewhat more evident gradient of representation of the radial digits laterally in M1 and the ulnar digits medially (Grafton et al., 1993; Kleinschmidt et al., 1997; Schieber, 1999). The greater finger independence of humans compared with monkeys thus results in part from differences in the passive biomechanical connections among tendons, in the organization of motor units and muscle 
bellies, and in the M1 hand representation. Nevertheless, in agreement with the observation that in purposeful movements humans rarely if ever need to move each finger independent of the others, even human finger movements are not completely independent.

\section{REFERENCES}

Adam A, De Luca CJ, Erim Z (1998) Hand dominance and motor unit firing behavior. J Neurophysiol 80:1373-1382.

Annett M (1992) Five tests of hand skill. Cortex 28:583-600.

Austin GJ, Leslie BM, Ruby LK (1989) Variations of the flexor digitorum superficialis of the small finger. J Hand Surg [Am] 14:262-267.

Bremner FD, Baker JR, Stephens JA (1991a) Correlation between the discharges of motor units recorded from the same and from different finger muscles in man. J Physiol (Lond) 432:355-380.

Bremner FD, Baker JR, Stephens JA (1991b) Variation in the degree of synchronization exhibited by motor units lying in different finger muscles in man. J Physiol (Lond) 432:381-399.

Buys EJ, Lemon RN, Mantel GW, Muir RB (1986) Selective facilitation of different hand muscles by single corticospinal neurones in the conscious monkey. J Physiol (Lond) 381:529-549.

Dassonville P, Zhu XH, Ugurbil K, Kim SG, Ashe J (1997) Functional activation in motor cortex reflects the direction and the degree of handedness. Proc Natl Acad Sci USA 94:14015-14018.

Engel KC, Flanders M, Soechting JF (1997) Anticipatory and sequential motor control in piano playing. Exp Brain Res 113:189-199.

Fahrer M (1981) Interdependent and independent actions of the fingers. In: The hand (Tubania R, ed), pp 399-403. Philadelphia: Saunders.

Fetz EE, Cheney PD (1980) Postspike facilitation of forelimb muscle activity by primate corticomotoneuronal cells. J Neurophysiol 44: 751-772.

Fetz EE, Perlmutter SI, Maier MA, Flament D, Fortier PA (1996) Response patterns and postspike effects of premotor neurons in cervical spinal cord of behaving monkeys. Can J Physiol Pharmacol 74:531-546.

Fish J, Soechting JF (1992) Synergistic finger movements in a skilled motor task. Exp Brain Res 91:327-334.

Fritz N, Schmidt C, Yamaguchi T (1992) Biomechanical organization of single motor units in two multi-tendoned muscles of the cat distal forelimb. Exp Brain Res 88:411-421.

Gonzalez MH, Whittum J, Kogan M, Weinzweig N (1997) Variations of the flexor digitorum superficialis tendon of the little finger. J Hand Surg [Br] 22B:277-280.

Grafton ST, Woods RP, Mazziotta JC (1993) Within-arm somatotopy in human motor areas determined by positron emission tomography imaging of cerebral blood flow. Exp Brain Res 95:172-176.

Hager-Ross CK, Schieber MH (1999) Human finger movements are not independent. Soc Neurosci Abstr 25:1915.

Hayes R, Schieber MH (1996) Human individuated finger movements: how independent are the digits? Soc Neurosci Abstr 22:426.

Humphrey DR, Reed DJ (1983) Separate cortical systems for control of joint movement and joint stiffness: reciprocal activation and coactivation of antagonist muscles. Adv Neurol 39:347-372.

Hunter JM (1990) Rehabilitation of the hand: surgery and therapy. St. Louis: Mosby.

Jancke L (1996) The hand performance test with a modified time limit instruction enables the examination of hand performance asymmetries in adults. Percept Mot Skills 82:735-738.

Kawashima R, Inoue K, Sugiura M, Okada K, Ogawa A, Fukuda H (1999) A positron emission tomography study of self-paced finger movements at different frequencies. Neuroscience 92:107-112.

Keen DA, Fuglevand AJ (1999) Inter-tendonous connections play a minor role in the broad distribution of motor unit force in extensor digitorum. Soc Neurosci Abstr 25:1149.

Kilbreath SL, Gandevia SC (1994) Limited independent flexion of the thumb and fingers in human subjects. J Physiol (Lond) 479:487-497.

Kimura D, Vanderwolf CH (1970) The relation between hand preference and the performance of individual finger movements by left and right hands. Brain 93:769-774.

Kleinschmidt A, Nitschke MF, Frahm J (1997) Somatotopy in the human motor cortex hand area. A high-resolution functional MRI study. Eur J Neurosci 9:2178-2186.

Linburg RM, Comstock BE (1979) Anomalous tendon slips from the flexor pollicis longus to the flexor digitorum profundus. J Hand Surg [Am] 4:79-83.

McKiernan BJ, Marcario JK, Karrer JH, Cheney PD (1998) Corticomotoneuronal postspike effects in shoulder, elbow, wrist, digit, and intrinsic hand muscles during a reach and prehension task. J Neurophysiol 80:1961-1980.
Mewes K, Cheney PD (1991) Facilitation and suppression of wrist and digit muscles from single rubromotoneuronal cells in the awake monkey. J Neurophysiol 66:1965-1977.

Mewes K, Cheney PD (1994) Primate rubromotoneuronal cells: parametric relations and contribution to wrist movement. J Neurophysiol 72:14-30.

Oldfield RC (1971) The assessment and analysis of handedness: the Edinburgh inventory. Neuropsychologia 9:97-113.

Parlow S (1978) Differential finger movements and hand preference. Cortex 14:608-611.

Perlmutter SI, Maier MA, Fetz EE (1998) Activity of spinal interneurons and their effects on forearm muscles during voluntary wrist movements in the monkey. J Neurophysiol 80:2475-2494.

Poliakov AV, Schieber MH (1999) Limited functional grouping of neurons in the primary motor cortex hand area during individuated finger movements: a cluster analysis. J Neurophysiol 82:3488-3506.

Rao SM, Bandettini PA, Binder JR, Bobholz JA, Hammeke TA, Stein EA, Hyde JS (1996) Relationship between finger movement rate and functional magnetic resonance signal change in human primary motor cortex. J Cereb Blood Flow Metab 16:1250-1254.

Remy P, Zilbovicius M, Leroy-Willig A, Syrota A, Samson Y (1994) Movement- and task-related activations of motor cortical areas: a positron emission tomographic study. Ann Neurol 36:19-26.

Sadato N, Ibañez V, Deiber MP, Campbell G, Leonardo M, Hallett M (1996) Frequency-dependent changes of regional cerebral blood flow during finger movements. J Cereb Blood Flow Metab 16:23-33.

Sanes JN, Donoghue JP, Thangaraj V, Edelman RR, Warach S (1995) Shared neural substrates controlling hand movements in human motor cortex [see comments]. Science 268:1775-1777.

Santello M, Soechting JF (1998) Gradual molding of the hand to object contours. J Neurophysiol 79:1307-1320.

Santello M, Flanders M, Soechting JF (1998) Postural hand synergies for tool use. J Neurosci 18:10105-10115.

Schieber MH (1990) How might the motor cortex individuate movements? Trends Neurosci 13:440-445.

Schieber MH (1991) Individuated finger movements of rhesus monkeys: a means of quantifying the independence of the digits. J Neurophysiol 65:1381-1391.

Schieber MH (1999) Somatotopic gradients in the distributed organization of the human primary motor cortex hand area: evidence from small infarcts. Exp Brain Res 128:139-148.

Schieber MH, Hibbard LS (1993) How somatotopic is the motor cortex hand area? Science 261:489-492.

Schieber MH, Poliakov AV (1998) Partial inactivation of the primary motor cortex hand area: effects on individuated finger movements. J Neurosci 18:9038-9054.

Schieber MH, Chua M, Petit J, Hunt CC (1997) Tension distribution of single motor units in multitendoned muscles: comparison of a homologous digit muscle in cats and monkeys. J Neurosci 17:1734-1747.

Schlaug G, Sanes JN, Thangaraj V, Darby DG, Jäncke L, Edelman RR, Warach S (1996) Cerebral activation covaries with movement rate. NeuroReport 7:879-883.

Schmied A, Vedel JP, Pagni S (1994) Human spinal lateralization assessed from motoneurone synchronization: dependence on handedness and motor unit type. J Physiol (Lond) 480:369-387.

Semmler JG, Nordstrom MA (1998) Hemispheric differences in motor cortex excitability during a simple index finger abduction task in humans. J Neurophysiol 79:1246-1254

Serlin DM, Schieber MH (1993) Morphologic regions of the multitendoned extrinsic finger muscles in the monkey forearm. Acta Anat (Basel) 146:255-266.

Soechting JF, Flanders M (1992) Organization of sequential typing movements. J Neurophysiol 67:1275-1290.

Triggs WJ, Subramanium B, Rossi F (1999) Hand preference and transcranial magnetic stimulation asymmetry of cortical motor representation. Brain Res 835:324-329.

von Schroeder HP, Botte MJ (1993) The functional significance of the long extensors and juncturae tendinum in finger extension. J Hand Surg [Am] 18:641-647.

von Schroeder HP, Botte MJ, Gellman H (1990) Anatomy of the juncturae tendinum of the hand. J Hand Surg [Am] 15:595-602.

Wessel K, Zeffiro T, Toro C, Hallett M (1997) Self-paced versus metronome-paced finger movements. A positron emission tomography study. J Neuroimaging 7:145-151.

Zatsiorsky VM, Li ZM, Latash ML (1998) Coordinated force production in multi-finger tasks: finger interaction and neural network modeling. Biol Cybern 79:139-150. 